Ann. Zootech., I972, 21 (3), 443-449.

\title{
INFLUENGE DE LA FINESSE DE HACHAGE DES ENSILAGES DE GRAMINÉES SUR LE COMPORTEMENT ALIMENTAIRE DES MOUTONS
}

\author{
J.-P. DULPHY et C. DEMARQUILLY \\ avec la collaboration technique de J.-M. Borssau. H. Bousguet et L. L'Hotelier \\ Station de Recherches sur l'Élevage des Ruminants, \\ Centre de Recherches de Clermont-Ferrand, I. N. R. A., \\ 63 - Saint-Genès-Champanelle
}

\section{RÉSUMÉ}

I. Nous avons comparé le comportement alimentaire de moutons recevant huit ensilages et un foin de fétuque élevée. Quatre des ensilages étaient hachés avant la mise en silo; parmi les quatre autres nous en avons haché deux à la sortie du silo.

2. Le hachage avant la mise en silo a permis une augmentation nette des quantités ingérées, de la durée journalière d'ingestion et du nombre de repas, mais faible des quantités ingérées lors des repas suivant la distribution de l'ensilage. Il a abaissé de façon importante la durée unitaire d'ingestion.

3. Le hachage à la sortie du silo de deux ensilages à brins longs a aussi permis une augmentation des quantités ingérées, quoique dans une proportion plus faible; la durée journalière d'ingestion n'a pas varié tandis que le nombre de repas a augmenté légèrement et la durée unitaire d'ingestion a diminué de façon importante.

4. L'amélioration des quantités ingérées par la finesse de hachage avant la mise en silo est due à la fois à l'amélioration de la qualité de conservation et au mode de présentation. Le mode d'action de ces deux phánomènes est discuté.

\section{INTRODUCTION}

Depuis r 965 des études se poursuivent au C. R. Z. V. de Theix (DEMARquiliy et JARRIGE, I970) sur la valeur alimentaire des ensilages de plantes fourragères. Dans ce cadre, nous enregistrons les manifestations du comportement alimentaire et mérycique des moutons (DULPHY, I97I et 1972) afin d'essayer d'interpréter 
les différences importantes de quantités ingérées observées entre les ensilages. C'est ainsi que nous avons plus particulièrement suivi les manifestations du comportement alimentaire des 6 moutons qui ont été utilisés pour mesurer la digestibilité et 1'ingestibilité (quantité de matière sèche ingérée) de 8 ensilages de fétuque élevée récoltés, soit avec une machine à fléaux (brins longs), soit avec une machine à tambour hacheur (brins courts) (cf. DulPhy et Demarquilily, I972).

\section{MATÉRIEL, ET MÉTHODES}

Les 8 ensilages ont été préparés à partir de deux variétés de fétuque élevée ( $L$ udion et $L P$ ) récoltées après le début de l'épiaison en juin 1970 . La récolte a été effectuée, soit avec une machine à couteaux (brins courts), soit avec une machine à fléaux (brins longs). Les fourrages ont été conservés dans des silos de $4 \mathrm{~m}^{3}$, en bois gainés de plastique (non hermétiques) ou en butyl (hermétiques), avec ou sans conservateur (acide formique à la dose de I,5 litre d'acide du commerce à 80 p. Too par tonne de matière fraîche). Les durées des périodes de distribution aux animaux et leur séquence ainsi que les caractéristiques de ces ensilages sont rapportées dans le tableau $\mathrm{I}$. Le passage d'un fourrage à un autre a été fait sans transition. En outre, un foin a été préparé à partir de la fétuque $L P$ et les deux ensilages de fétuque $L$ udion récoltés avec la machine à fléaux ont également été distribués après un hachage fin dans un hache-paille à la sortie du silo.

Un seul lot de 6 béliers castrés de race Texel a servi à cette étude. Les quantités ingérées par les animaux ont été mesurées chaque jour pour une ditribution ad libitum ( Io à I 5 p. Ioo de refus) en 2 repas à $8 \mathrm{~h}$ et à $\mathrm{I} 7 \mathrm{~h}$. La mesure des quantités offertes a été faite deux fois par jour, à chaque distribution, et celle des refus une fois. Les quantités ingérées lors des deux repas suivant une distribution de fourrage ont été estimées en rapportant la durée de ces repas à la durée journalière d'ingestion; cette estimation introduit une certaine erreur qui augmente avec le nombre de repas (Dulphy, 1972) et est de l'ordre de 2 à $4 \mathrm{~g} / \mathrm{kg} \mathrm{P}^{0,75}$ compte tenu des contrôles effectués sur de nombreux ensilages durant l'hiver I97I-1972 (DULPHy, résultats non publiés).

Les manifestations du comportement alimentaire et mérycique des animaux ont été enregistrées graphiquement (RuckeBusch, I963) pendant les 3 premiers jours de chaque période et pendant les 5 derniers jours, les animaux pouvant être alors considérés comme bien adaptés au fourrage étudié.

\section{RÉSULTATS}

Les quantités ingérées par les animaux sont rapportées dans le tableau 2. Elles sont très différentes selon la machine de récolte utilisée : $53,4 \mathrm{~g} / \mathrm{kg} \mathrm{P} \mathrm{P}^{0,75}$ pour la machine à couteaux contre $26, \mathbf{I} \mathrm{g} / \mathrm{kg} \mathrm{P} \mathrm{P}^{0,75}$ pour la machine à fléaux ; 39,0 $\mathrm{g}$ pour les 2 ensilages hachés à la sortie du silo contre $25,8 \mathrm{~g}$ pour les ensilages correspondants non hachés. Des différences existent selon le type de fermentation induit par la présence ou non d'acide formique : 48,6 g avec acide contre $35,4 \mathrm{~g}$ sans acide.

Le comportement alimentaire des animaux pendant les 5 derniers jours de chaque période est résumé dans le tableau 2.

La durée journalière d'ingestion a été plus élevée pour les 4 ensilages à brins courts que pour les 4 ensilages à brins longs : en moyenne 215 contre 162 minutes. Cela doit résulter vraisemblablement de leur meilleure qualité de conservation et non de leur meilleure préhensibilité ; en effet, la durée journalière d'ingestion a augmenté significativement $(\mathrm{P}<0,05)$ avec la teneur en acide lactique des ensilages $(r=+0,72)$ alors qu'elle n'a pas varié pour les 2 ensilages à brins longs quand ils ont été hachés à la sortie du silo : I54 mn après hachage contre I64 avant. 


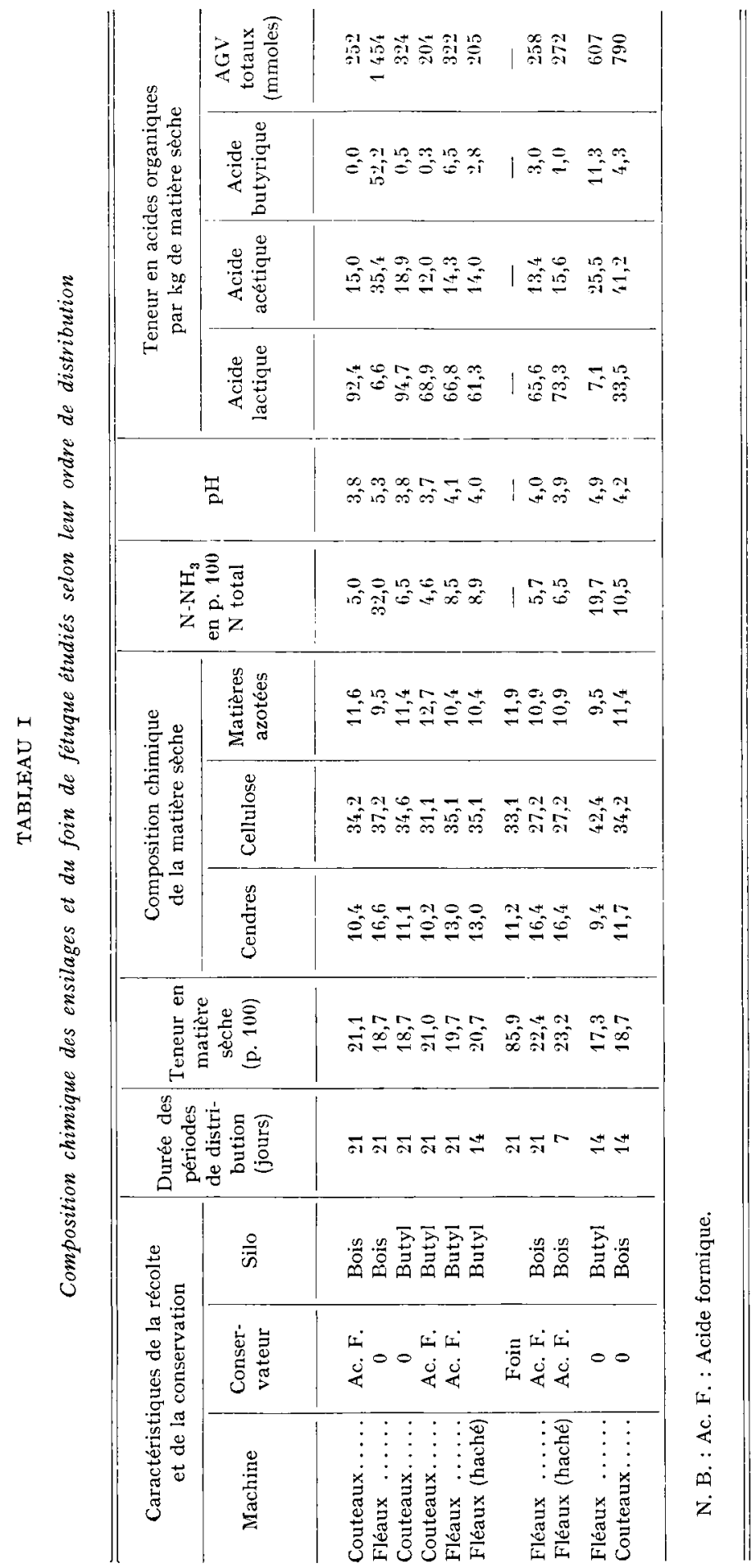


J.-P. DULPHY, C. DEMARQUILLY

\begin{tabular}{|c|c|c|c|c|c|}
\hline \multirow[b]{2}{*}{ 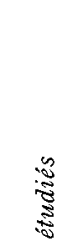 } & \multirow{2}{*}{ 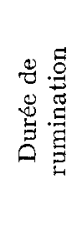 } & 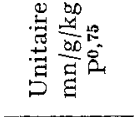 & 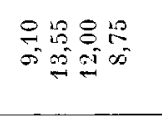 & 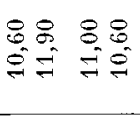 & 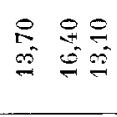 \\
\hline & & 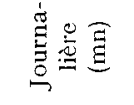 & 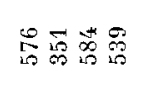 & 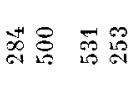 & 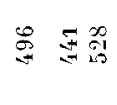 \\
\hline 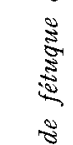 & \multirow{2}{*}{ 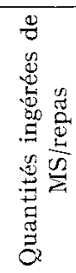 } & 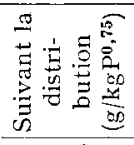 & 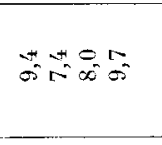 & 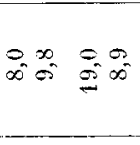 & $\overrightarrow{0}=0$ \\
\hline 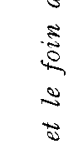 & & 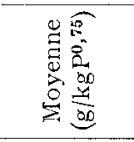 & 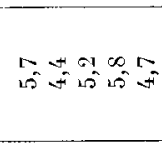 & $\begin{array}{ccc}0 & 0 & \infty \\
0 & -\infty & 10\end{array}$ & $\Rightarrow$ \\
\hline 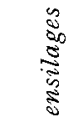 & \multirow{2}{*}{ 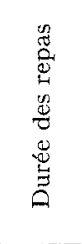 } & 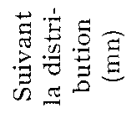 & 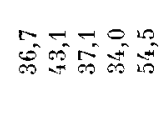 & 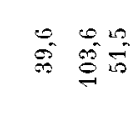 & 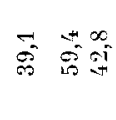 \\
\hline $\begin{array}{l}3 \\
3 \\
5 \\
0 \\
0 \\
01\end{array}$ & & 总总 & बi़ी & $=\frac{10}{20}$ & 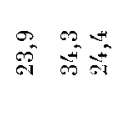 \\
\hline 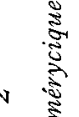 & \multicolumn{2}{|c|}{ 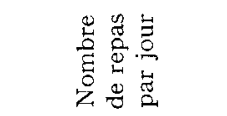 } & $\begin{array}{l}\Rightarrow=0 \\
=100\end{array}$ & 3 is 5 & of $8=$ \\
\hline 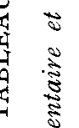 & \multirow{2}{*}{ 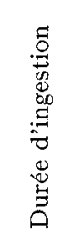 } & 总总 & 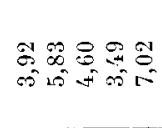 & 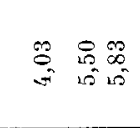 & 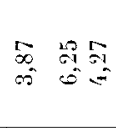 \\
\hline 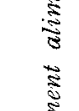 & & 氮总司 & $\underset{0}{\infty}$ & 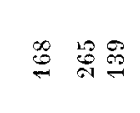 & 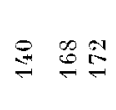 \\
\hline 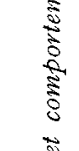 & \multicolumn{2}{|c|}{ 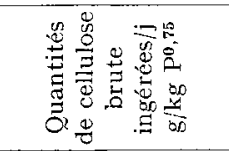 } & $\begin{array}{cc}0 & 0 \\
A & 0\end{array}$ & $\begin{array}{l}5 \\
=0 \\
=10\end{array}$ & $\begin{array}{l}\theta \\
\theta\end{array}$ \\
\hline 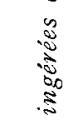 & \multicolumn{2}{|c|}{ 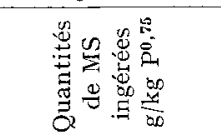 } & 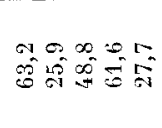 & 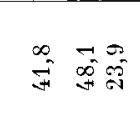 & 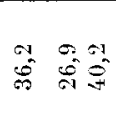 \\
\hline 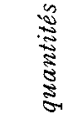 & \multicolumn{2}{|c|}{ 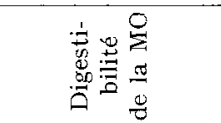 } & 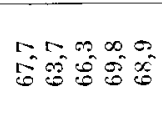 & $1 \begin{array}{l}0 \\
8 \\
80 \\
0\end{array}$ & $\begin{array}{lll}E & * \\
5 & 0 & 0\end{array}$ \\
\hline \multirow{3}{*}{ 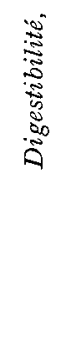 } & \multirow{3}{*}{ 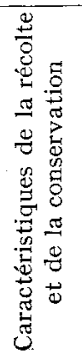 } & $\frac{0}{n}$ & 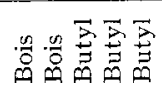 & $\underset{\vec{E}}{\bar{E}}$ & 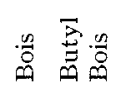 \\
\hline & & 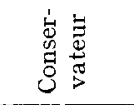 & 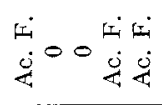 & 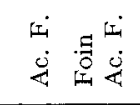 & 岴 \\
\hline & & 兽 & 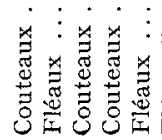 & 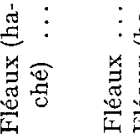 & 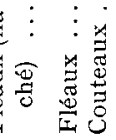 \\
\hline
\end{tabular}


En rèvanche, la durée unitaire d'ingestion (temps passé à ingérer I g/ $/ \mathrm{kg} \mathrm{P}^{\mathbf{0}, 75}$ ) qui représente l'inverse de la vitesse d'ingestion, a été plus faible pour les 4 ensilages à brins courts : 4,07 contre $6,23 \mathrm{mn} / \mathrm{g} / \mathrm{kg} \mathrm{P}^{0,75}$ en moyenne. Cela résulte directement du fait qu'ils ont été hachés finement puisque le hachage à la sortie du silo des ensilages à brins longs a diminué de 6,42 à $3,95 \mathrm{mn} / \mathrm{g} / \mathrm{kg} \mathrm{P} \mathrm{P}^{0,75}$ leur durée unitaire d'ingestion.

Le nombre de repas journaliers a été plus élevé pour les 4 ensilages à brins courts, mais cela est dû à la fois au hachage proprement dit et à leur meilleure qualité de conservation : $r=+0,62(\mathrm{P}<0$, IO) avec la teneur en acide lactique. Il est curieux de constater que, malgré des différences très importantes d'ingestibilité entre les ensilages, les quantités estimées de matière sèche ingérées lors des repas suivant les distributions on été peu variables d'un ensilage à $1^{\prime}$ autre $\left(7,4\right.$ à $\left.I 0,0 \mathrm{~g} / \mathrm{kg} \mathrm{P}^{0,75}\right)$, mais plus faibles que celles ingérées sous forme de foin ( $\mathrm{r} 9, \mathrm{O} \mathrm{g}$ ). Les quantités journalières d'ensilage ingérées dépendent alors essentiellement de ce qui est ingéré en dehors des repas suivant les distributions et, par là, du nombre de repas ( $r=+0,9^{* *}, n=8$ données) et, dans une moindre mesure, de la durée journalière d'ingestion $\left(r=+0,89^{* *}\right)$ et de la durée unitaire d'ingestion $(r=+0,89$.

Quant à la rumination, nous pouvons noter que sa durée journalière dépend essentiellement de la quantité de matière sèche $\left(r=-0,84^{* *}\right)$ ou de cellulose brute $\left(r=+0,87^{* *}\right)$ ingérée mais que sa durée unitaire (temps passé à ruminer rapporté à la quantité de matière sèche ingérée par 24 heures) n'est pas reliée significativement aux quantités ingérées ou à la digestibilité des fourrages.

L'étude du comportement des animaux lors des 3 premiers jours de chaque période a permis de mettre en évidence que les différences de comportement (de même que les différences de quantités ingérées) suivant la finesse de hachage de l'ensilage distribué s'établissaient très rapidement, du moins pour les ensilages à brins longs et le foin qui, dès le premier jour, ont été offerts à volonté. En effet, pour ces fourrages, le niveau d'ingestion des moutons a été stabilisé dès le premier jour, sauf pour l'ensilage à brins longs dont l'étude s'est située juste après celle $\mathrm{du}$ foin et pour lequel la quantité ingérée a diminué progressivement de $38 \mathrm{p}$. Ioo pendant les 6 premiers jours avant de se stabiliser. Pour ces ensilages à brins longs la durée journalière d'ingestion, la durée unitaire d'ingestion et le nombre de repas ont été plus élevés le premier jour et, déjà à un moindre degré, le deuxième et le troisième jour de la distribution, que lorsque les animaux ont été adaptés : respectivement pour le premier jour 202 contre 162 minutes lorsque les animaux ont été adaptés, 7,48 contre $6,23 \mathrm{mn} / \mathrm{g} / \mathrm{kg} \mathrm{P}^{0,75}$ et 7,8 contre 5,2 . Il en est de même avec le foin.

\section{DISCUSSION}

En augmentant soit directement, soit indirectement par l'intermédiaire de l'amélioration de la qualité de la conservation, la durée journalière d'ingestion, la vitesse d'ingestion et le nombre de repas journaliers, le hachage avant la mise en silo entraîne une augmentation importante des quantités d'ensilage ingérées alors qu'il a peu ou pas d'action sur la quantité de foin ou de fourrage vert non ingérée (DuLPHy, résultats publiés). 
S'il est possible d'expliquer l'influence du hachage sur la vitesse d'ingestion par suite d'une meilleure préhensibilité des ensilages hachés finement, il est beaucoup plus difficile d'expliquer son influence et celle de la qualité de la conservation, sur la durée journalière d'ingestion et le nombre journalier de repas.

Comme dans d'autres essais (cf. Dulphy et Demarquilly, I97I) nous observons que les moutons recevant des ensilages sont rapidement rassasiés; lors des repas faisant suite aux distributions, ils s'arrêtent de manger après avoir ingéré une quantité faible (environ $9 \mathrm{~g} / \mathrm{kg} \mathrm{P}^{\mathbf{O}^{, 75}}$ ) et peu variable d'un ensilage à l'autre, mais une quantité très variable de métabolites fermentaires ( $\mathrm{I}, 98$ à $10.75 \mathrm{~m} / \mathrm{moles}$ d'AGV par $\mathrm{kg}$ de poids métabolique, 49 à $867 \mathrm{mg}$ d'acide lactique par $\mathrm{kg}$ de poids métabolique)puisque les caractéristiques fermentaires des ensilages étudiés sont très différentes. L'ingestion ne semble donc pas arrêtée par les métabolites apportés par les ensilages mais, soit par ceux résultant de leur digestion (ceuxci ne doivent pas être très différents suivant les ensilages puisqu'ils sont tous issus des mêmes plantes sur pied), soit peut-être par suite d'un encombrement rapide du rumen au niveau du cardia. En effet, on peut supposer que, par suite de la densité élevée des ensilages due au tassement auquel ils ont été soumis dans les silos, et peut-être aussi de la structure du contenu du rumen (stratification et longueur des particules, qui expliqueraient que lors du passage d'un foin à un ensilage la quantité d'ensilage ingérée diminue progressivement avant de se stabiliser), les bols alimentaires d'ensilage s'accumulent à proximité du cardia.

$\mathrm{Si}$ elle était vérifiée, cette dernière hypothèse permettrait en outre d'expliquer le nombre de repas plus élevé observé avec les ensilages hachés. En effet, la dispersion des bols alimentaires et leur homogénéisation au contenu du rumen doivent être beaucoup plus rapide qu'avec les ensilages à brins longs; les repas peuvent donc être plus nombreux et la quantité journalière ingérée plus importante. En outre, le hachage doit accélérer la vitesse de digestion dans le rumen en augmentant la surface d'attaque. Cependant les ensilages hachés avant la mise en silo sont ingérés en quantité plus élevée que ceux qui ne le sont qu'à la sortie du silo. La qualité de la conservation joue donc aussi un rôle important qui s'exerce par l'intermédiaire de la durée journalière d'ingestion et du nombre de repas. Il est difficile de savoir si les métabolites fermentaires apportés par l'ensilage agissent sur ces 2 paramètres en modifiant la motricité du rumen, la vitesse de digestion de l'ensilage dans le rumen ou directement l'appétit de l'animal. La première hypothèse semble cependant la plus vraisemblable et nous nous efforcerons de la vérifier ultérieurement.

Reçu pour publication en mars $19 \% 2$.

\section{SUMMMARY}

\section{EFFECT OF CHOPPING FINENESS OF GRASS SILAGES}

ON THE FEEDING BEHAVIOUR OF SHEEP

t. A comparative study was made on the feeding behaviour of sheep offered eight silages and a tall fescue hay. Four of the silages were finely chopped before ensiling; of the four other lacerated silages $(10-20 \mathrm{~cm}$ particle length), two were finely chopped after ensiling.

2. Fine chopping before ensiling enabled a definite increase in the total voluntary feed intake, daily time spent eating and number of meals, but only a slight increase in the voluntary 
intake at the two principal daily meals which followed after fresh silage had been offered. The rate of eating $\left(\mathrm{mn} / \mathrm{g} / \mathrm{kg} \mathrm{P}^{\mathbf{0 . 7 5}}\right)$ was widely lowered by fine chopping.

3. Chopping after ensiling of two lacerated silages also produced a higher voluntary intake, which was however lower than that obtained when chopping was carried out before ensiling. The daily time spent eating did not vary whereas the number of meals slightly increased and the rate of eating (in $\mathrm{mn} / \mathrm{g} / \mathrm{kg} \mathrm{P}^{\mathbf{0 . 7 5}}$ ) considerably decreased.

4. The improvement of the voluntary feed intake due to chopping depends both on the improvement in the quality of conservation and on the physical form of the forage. The mode of action of these two phenomena is discussed.

\section{RÉFÉRENCES BIBIOGRAPHIQUES}

Demarquilly C., Jarrige R., I97o. The effect of method of forage conservation on digestibility and voluntary intake. Proc. XIth Int. Grassland Congress, 733-737.

Dulphy J.-P., I97I. Influence du poids vif et du niveau d'ingestion sur le comportement alimentaire et mérycique du mouton. Ann. Zootech., 20, 477-486.

Dulphy J.-P., I972. Étude de quelques relations entre le mode de conservation du fourrage ingéré et le comportement alimentaire et mérycique des moutons. Ann. Zootech. 21, 429-442.

Dulphy J.-P., Demarguilly C., 1971. Liaisons entre le comportement des moutons pendant l'ingestion et les quantités de fourrages ingérées. Xe Congrès Int. de Zootechnie (Nutrition ruminants).

Dulphy J.-P., Demarouilly C., I972. Influence de la machine de récolte sur la valeur alimentaire des ensilages. Résultats préliminaires. Ann. Zootech., 21, 162-173.

RuckebUSCH Y., I963. Recherches sur la régulation centrale ducomportement alimentaire des ruminants. Thèse Doct. Sci. Nat., Lyon. 